\title{
Executive Function, Eating Behavior, and Preoperative Weight Loss in Bariatric Surgery Candidates: An Observational Study
}

\author{
Gro Walø-Syversen $^{a} \quad$ Ingela L. Kvalem ${ }^{b}$ Jon Kristinsson ${ }^{c} \quad$ Inger L. Eribe ${ }^{c}$ \\ Øyvind $\mathrm{R}^{\mathrm{a}} \mathrm{d}$ Camilla Lindvall Dahlgren ${ }^{\mathrm{a}}$ \\ a Regional Department for Eating Disorders, Oslo University Hospital, Oslo, Norway; \\ ${ }^{b}$ Department of Psychology, University of Oslo, Oslo, Norway; ${ }^{c}$ Centre for Morbid Obesity \\ and Bariatric Surgery, Oslo University Hospital, Oslo, Norway; ${ }^{d}$ Institute of Clinical Medicine, \\ Faculty of Medicine, University of Oslo, Oslo, Norway
}

\section{Keywords}

Bariatric surgery · Executive function · Eating behavior · Preoperative weight loss

\begin{abstract}
Background: Individual differences in executive function may influence eating behavior, weight loss (WL), and WL maintenance in obesity treatment. Executive function, which designates top-down cognitive control processes, has been related to eating behaviors which may impact weight, and has been found to be predictive of WL in both behavioral WL programs and after bariatric surgery. Currently, we lack knowledge on the role of executive function in the period before bariatric surgery. If executive function impacts eating behavior and WL in the preoperative period, it may be a target for clinical attention in this stage. Objectives: We aimed to examine the relationship between objective performance-based measures of executive function, eating patterns, and WL in the preoperative period. Method: Baseline data in an ongoing observational longitudinal study of bariatric surgery patients were used. Eighty patients completed neuropsychological testing and self-report questionnaires 4 weeks prior to surgery. Results: We found that working memory predicted WL before surgery and inhibitory control predicted adherence to dietary recommendations. Conclusion: Our study indicates that executive function may play a role in short-term WL and dietary adherence prior to surgery, suggesting that executive function in the preoperative period deserves an extended research focus.


Walø-Syversen et al.: Bariatric Surgery Candidates: Executive Function, Eating Behavior, and Preoperative WL

\section{Introduction}

Severe obesity (body mass index $[\mathrm{BMI}] \geq 40$ ) is a multifactorial disease that has been linked to reduced neuropsychological functioning in several cognitive domains [1-3] and especially in the area of executive function $[1,2,4-6]$. Executive function is involved in health outcomes across various chronic medical conditions [7] and there is reason to consider the impact of executive function in obesity treatment $[5,8]$. For example, executive function has been related to various eating behaviors such as loss-of-control (LOC) eating [9] and the intake of high caloric food [8], both associated with weight gain. Furthermore, executive function has been found to be predictive of treatment adherence [10] and WL, in both behavioral WL programs [11,12] and after bariatric surgery [13-15]. Currently, we lack knowledge on the role of executive function in the period before bariatric surgery when WL and dieting are required.

Executive function is a broad neuropsychological concept referring to several subdomains of top-down regulatory processes guiding goal-directed behavior [16]. Executive skills are essential to self-regulation and control of behavior. Such skills involve several core cognitive processes such as inhibition, shifting, and working memory $[17,18]$. In obesity and bariatric surgery samples, numerous reports suggest a reduced performance in the neuropsychological tests that measure different aspects of executive function $[1-3,9,19-24]$ including both inhibitory control $[20,25]$ and working memory $[26,27]$. The comorbid conditions that typically join with obesity are known to adversely affect neurocognitive outcomes [1]; however, executive function impairment appears to be less dependent of comorbidity [28].

The latest reviews $[5,8]$ and previous studies that examine executive function in eating behavior [29-31] and WL [15, 32] have primarily emphasized 2 suggested subcomponents of executive function $[17,18]$, namely working memory and inhibitory control. Most of the studies have focused on inhibitory control $[9,20,25]$, which refers to the capacity to purposely inhibit a dominant, automatic, internal predisposition or response [17]. Existing evidence points to difficulties in the maintenance of inhibitory control over time, as well as reduced inhibitory control in food-specific stimuli contexts [20]. Better inhibitory control is usually found to be related to a lower BMI [20] and a predictor of BMI and WL in behavioral WL programs $[11,25,32]$. Furthermore, a reduced inhibition capacity has been associated with a more pathological facet of eating behavior which is prevalent in bariatric surgery populations [33-35], namely binge-eating [9], and is also possibly related to LOC grazing [36].

A recent review concluded that executive function may especially be needed in situations requiring change in eating behavior and dieting [8]. In this regard, inhibitory control seems to be related to ceasing undesirable eating behavior and resisting impulses to eat unhealthy food, whereas working memory (which refers to retaining and mentally manipulating information) [17] appears to be associated with the planning and initiation of healthy eating behavior such as eating more vegetables and fruit $[5,8]$. In sum, executive function is predictive of BMI and WL in behavioral obesity treatment and has been related to eating behavior $[5,8]$. It therefore seems reasonable to assume that executive function would influence weight and eating patterns prior to bariatric surgery.

Patients undergoing bariatric surgery are advised to follow a low-calorie diet to achieve preoperative $\mathrm{WL}$, mainly to stabilize any preoperative medical comorbidities and enhance surgical outcomes [37]. We have scarce knowledge about the executive functions that influence WL and eating behavior prior to surgery and if they differ from those that contribute to successful postoperative outcomes. Executive dysfunction at a preoperative stage may be suggestive of postoperative difficulties in WL maintenance and treatment adherence. We already know that reduced executive function is present in bariatric surgery patients $[2,4$, $28,38,39]$. A number of studies from the Longitudinal Assessment of Bariatric Surgery 
Fig. 1. Flow chart of the recruit-

Walø-Syversen et al.: Bariatric Surgery Candidates: Executive Function, Eating Behavior, and Preoperative WL

ment process.

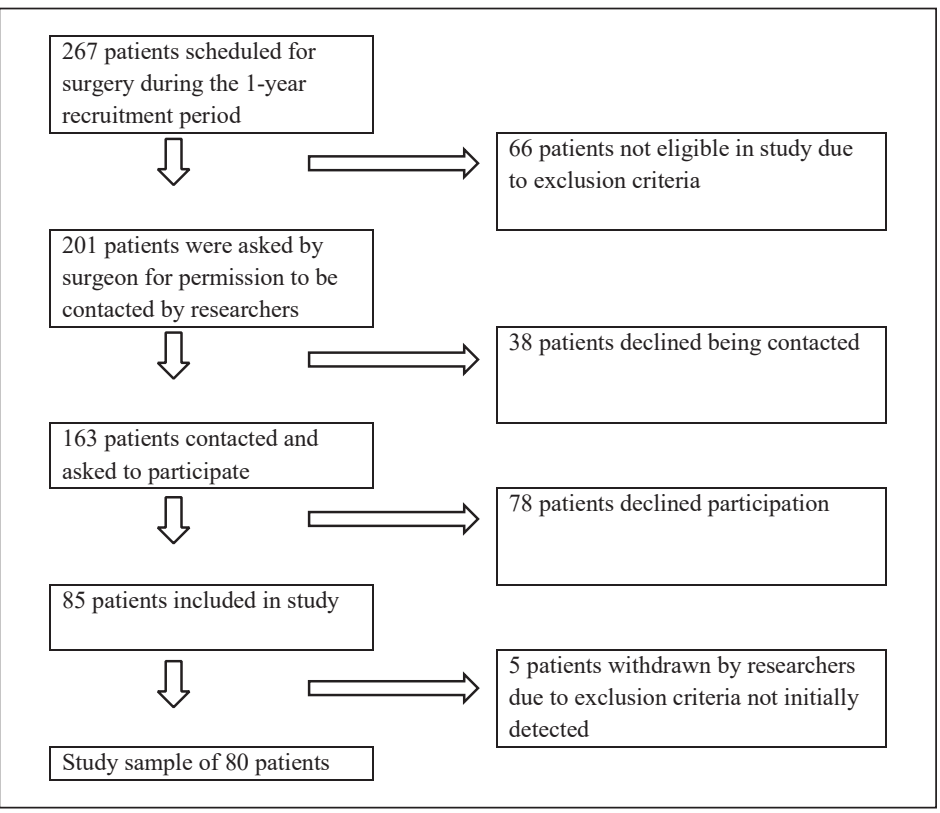

Consortia (LABS) have demonstrated that better preoperative executive performance predicts a lower BMI and a greater loss of excess weight 12 months after surgery $[13,14,40]$. Furthermore, preoperative executive performance has been related to treatment adherence as early as 6 weeks after surgery [10]. However, to our knowledge, no study has investigated the role of executive function in the period before surgery.

In this study, we first wished to examine the association of executive function with weight status (BMI) and WL in the preoperative period. We chose to include 4 executive function measures, 2 representing working memory and 2 for inhibitory control, as these are subcomponents that the literature suggests as relevant $[5,8]$. The measures of inhibitory control are also the most commonly used test in obesity and bariatric surgery samples [9, 20]. Second, we wished to investigate the relationship between executive function and eating behavior. A healthy diet is recommended in the period both prior to and after surgery and patients are advised to adhere to national dietary recommendations. In addition, we wished to explore 2 potentially pathological facets of eating behavior that could possibly deter WL and are commonly reported in bariatric surgery populations: binge-eating [41] and grazing [33]. Given that obesity has a strong link to depression [1,41], which is consistently associated with cognitive impairment [42], a measure to control for depression was included. Due to the inconsistency of results regarding medical comorbidities across previous studies [1], we also explored the influence of medical comorbidities on executive function [28].

We hypothesized that measures of executive function would be related to preoperative $\mathrm{BMI}$ and $\mathrm{WL}$, and that executive function would predict healthier eating patterns and less eating pathology.

\section{Materials and Methods}

\section{Study Design, Procedures, and Participants}

This study analyzes a subset of data from the baseline testing in an ongoing prospective longitudinal observational study, the Oslo Bariatric Surgery Study (OBSS) Cognitive, at the Centre for Morbid Obesity and Bariatric Surgery, Oslo University Hospital, Oslo, Norway. The 
Walø-Syversen et al.: Bariatric Surgery Candidates: Executive Function, Eating Behavior, and Preoperative WL

project is an extension of the OBSS which explores psychosocial predictors of behavior and weight change after bariatric surgery [43]. To be eligible for bariatric surgery in Norwegian public hospitals, patients need to present with morbid obesity (BMI $\geq 40$ ) or have a BMI $>35$ in combination with comorbidity. Furthermore, prior nonsurgical WL interventions should have been tried. All potential bariatric surgery candidates are required to be present at an obligatory 40-h course that focuses on different treatment interventions (surgical and nonsurgical), diet, exercise, and lifestyle changes before assessment by a multidisciplinary team for their eligibility. Patients are required to attend a course with a dietitian after the decision has been made to perform surgery. Three weeks prior to the surgery, patients are instructed to adhere to a diet of 1,200 calories a day. The study recruitment process is visualized in Figure 1. Exclusion criteria were: not understanding Norwegian, neurological disorder, moderate/severe head injury, past/ present history of severe psychiatric illness, past/current alcohol or drug abuse/dependence, history of learning disorder, developmental disability, and impaired sensory function. The neuropsychological assessment was scheduled for 30 days ( \pm 5 days) before the operation and prior to the preoperative diet. The final sample $(n=80)$ comprised 61 females and 19 males who underwent gastric sleeve (51\%), gastric bypass (50\%), mini gastric bypass $(13 \%)$, or duodenal switch $(1 \%)$ surgery. Compared to the total number of patients scheduled for surgery in the recruitment period $(n=267)$, results revealed no significant differences between participants and nonparticipants in terms of age or male-to-female ratio.

\section{Measures}

Somatic Health Measures and Definition of Comorbidities

Participants' weight (lightly dressed and without shoes) was measured on a platform scale SECA 635 Class III. Blood pressure was measured (CAS 730 Monitor, Ortomodic AS) with the cuff placed around the upper arm while the patient was sitting and resting for a minimum of $3 \mathrm{~min}$. Hypertension was defined as having a systolic blood pressure of $\geq 140 \mathrm{~mm}$ $\mathrm{Hg}$ or a diastolic blood pressure of $\geq 90 \mathrm{~mm} \mathrm{Hg}$ during testing or self-reported use of antihypertensive medication on the day of testing. Reporting of diabetes, sleep apnea, and dyslipidemia were based on diagnoses from patients' medical records.

\section{Self-Report Questionnaires}

Adherence to dietary recommendations is a 6-item self-report measure [44] based on the dietary guidelines from the Norwegian Health Directorate [45], asking to what degree $(0=$ not at all and $7=\mathrm{a}$ lot) the recommendations were followed during the last 4 weeks before testing (e.g., I limit my intake of fat and sugar; I choose low-fat dairy products). A composite average score was calculated, where a higher score indicates greater adherence. Cronbach's $\alpha$ for the scale was 0.78 .

Binge-eating problems were assessed by using 3 items from the Eating Disorder Examination Questionnaire (EDE-Q) [46, 47]: frequency of binge-eating last 28 days, frequency of LOC binge-eating and the number of days with LOC binge-eating. We used the frequency of LOC binge-eating in the analyses.

Grazing was assessed by means of the Repetitive Eating Questionnaire (Rep[eat]) [35], a 12 -item self-report questionnaire asking for the frequency $(0=$ never and $6=$ more than once every day) of grazing behaviors and attitudes [48]. Participants indicated eating behaviors the last 4 weeks before testing. The questionnaire yields 3 scoring options, a total scale average, a compulsive grazing mean score, and a repetitive eating mean score. A higher score indicates a higher frequency. We used total average score in the analyses. Cronbach's $\alpha$ for the total scale was 0.93 .

Depressive symptoms were assessed using the Depression Subscale from the Hospital Anxiety and Depression Scale (HADS) [49]. Seven items (response range 0-3) were summed 
Walø-Syversen et al.: Bariatric Surgery Candidates: Executive Function, Eating Behavior, and Preoperative WL

providing a score in the range from $0-21$. A score of $\leq 7$ is usually identified as a noncase, whereas scores of 8-11, 11-14, and 15-21 designate the presence of mild, moderate, and severe depressive symptoms, respectively [50]. The Cronbach's $\alpha$ for the subscale was 0.76 .

Neuropsychological Tests

Two subscales, Matrix Reasoning and Vocabulary, from the Wechsler Adult Intelligence Scale (WAIS-IV) were used to control for intelligence [51, 52].

\section{Inhibitory Control}

The Delis Kaplan Executive Function System (D-KEFS) Color-Word Interference Test (CWIT) [53], based on the classical Stroop test, was used. The outcome measures were: total errors and total time in the incongruent color-word condition (scaled score). A higher score indicates a better performance.

The Stop Signal Task (CANTAB) [54] measures the ability to inhibit a prepotent inappropriate motor reaction in response to a stop signal [55]. We used the stop signal reaction time (SSRT) as outcome measure (i.e., the latency in milliseconds participants need to internally suppress a motor response). A longer latency indicates a poorer performance.

\section{Working Memory}

Spatial Working Memory (SWM) (CANTAB) [54] is a measure assessing the ability to retain and manipulate spatial information in working memory. We used between errors as outcome measure (the total number of times the participant revisits a box where a token was previously found). A higher score indicates a lower performance.

One Touch Stockings of Cambridge (OTS) (CANTAB) [54] is a measure of spatial planning and working memory. The outcome measure was the number of problems solved on the first choice. A higher score indicates a better performance.

\section{Statistical Analyses}

Data Screening and Preparation

All variables were screened for violations of the assumptions relevant to each of the statistical analyses performed. The presence of outliers was assessed, and their influence reduced. One variable (binge-eating frequency) was inverse-transformed due to severe skewness; original values are reported due to ease of interpretation. There were $<5 \%$ missing values per self-report questionnaire and 1 case with 1 missing neuropsychological test (OTS) score. Ipsativ mean substitution (self-report forms) and pairwise deletion (3 nonresponse cases on the bingeeating questions and 1 missing neuropsychological test score) were used to handle missing data.

\section{Analyses}

Descriptive statistics, independent-samples $t$ test, and $\chi^{2}$ test with the Yates continuity correction were performed to describe the sample. A series of preliminary two-way betweengroups analysis of covariance were conducted to examine the effect of medical comorbidities and gender on neuropsychological test performance, with age included as a covariate. Preliminary analyses using bivariate correlations were conducted to examine relationships between executive function tests, weight and eating behavior, and control variables. Relevant variables that were significantly correlated were included in 2 successive separate hierarchical multiple-regression analyses, while controlling for gender, age (only for tests without ageadjusted scores), depression, and estimated intelligence. To reduce the number of predictors only Vocabulary was used as an estimate of intelligence. Somatic disorders that significantly influenced the neuropsychological tests results were planned to be included in the primary regression models as control variables. 
Walø-Syversen et al.: Bariatric Surgery Candidates: Executive Function, Eating Behavior, and Preoperative WL

Table 1. Descriptive statistics for the continuous study variables

\begin{tabular}{|c|c|c|c|c|c|c|c|c|c|}
\hline & \multicolumn{3}{|c|}{ Total $(n=80)$} & \multicolumn{2}{|c|}{ Females $(n=61)$} & \multicolumn{4}{|c|}{ Males $(n=19)$} \\
\hline & mean & SD & range & mean & SD & mean & SD & $t$ value & $p$ value \\
\hline \multicolumn{10}{|l|}{ BMI and weight loss } \\
\hline BMI at test & 43.7 & 4.92 & $34.6-56.0$ & 43.5 & 5.06 & 44.3 & 4.50 & -0.64 & 0.521 \\
\hline 30-Day preoperative WL (\%) & 2.9 & 3.2 & -3.51 to 9.63 & 2.92 & 2.96 & 2.72 & 2.88 & -0.25 & 0.803 \\
\hline \multicolumn{10}{|l|}{ Eating behavior } \\
\hline Dietary adherence & 4.76 & 1.24 & $1-7$ & 4.87 & 1.16 & 4.41 & 1.45 & 1.42 & 0.158 \\
\hline Grazing (Rep[eat] total mean score) & 1.21 & 1.09 & $0-4.73$ & 1.27 & 1.06 & 1.01 & 1.19 & -0.90 & 0.368 \\
\hline LOC binge-eating (frequency) & 1.35 & 4.39 & $0-30$ & 1.45 & 4.94 & 1.05 & 1.95 & -0.72 & 0.736 \\
\hline \multicolumn{10}{|l|}{ Neuropsychological test scores } \\
\hline Working memory (SWM errors) & 39.2 & 20.2 & $3-88$ & 38.3 & 19.5 & 42.1 & 22.8 & -0.71 & 0.480 \\
\hline $\begin{array}{l}\text { Planning (OTS problems solved } \\
\text { on first choice) }\end{array}$ & 10.6 & 2.22 & $5-15$ & 10.6 & 2.26 & 10.6 & 2.16 & 0.00 & 0.998 \\
\hline $\begin{array}{l}\text { Response inhibition } \\
\text { (SSRT stop latency) }\end{array}$ & 200 & 46.5 & $123-332$ & 196 & 45.9 & 210 & 48.5 & -1.06 & 0.291 \\
\hline \multicolumn{10}{|l|}{ Inhibitory control (CWIT) } \\
\hline $\begin{array}{l}\text { Total error inhibition trial } \\
\text { (scaled score) }\end{array}$ & 10.5 & 1.88 & $6-13$ & 10.5 & 1.84 & 10.4 & 3.67 & 0.59 & 0.553 \\
\hline $\begin{array}{l}\text { Total time inhibition trial } \\
\text { (scaled score) }\end{array}$ & 9.90 & 2.87 & $1-15$ & 10.2 & 2.5 & 8.79 & 3.94 & 1.96 & 0.053 \\
\hline \multicolumn{10}{|l|}{ Control variables } \\
\hline Age & 41.0 & 10.7 & $19-62$ & 40.0 & 11.1 & 44.5 & 9.03 & -1.60 & 0.112 \\
\hline Matrix reasoning & 10.8 & 3.65 & $1-21$ & 11.2 & 3.50 & 9.63 & 3.94 & 1.72 & 0.089 \\
\hline Vocabulary & 8.0 & 1.81 & $3-13$ & 8.16 & 1.65 & 7.74 & 2.25 & 0.89 & 0.372 \\
\hline Depression & 3.95 & 3.37 & $0-13$ & 4.02 & 3.47 & 3.73 & 3.10 & 0.32 & 0.746 \\
\hline
\end{tabular}

BMI, body mass index; WL, weight loss; Rep(eat), repetitive eating questionnaire; LOC, loss-of-control; SWM, spatial working memory; OTS, One Touch Stockings of Cambridge; SSRT, stop signal reaction time; CWIT, Color-Word Interference Test.

\section{Results}

\section{Sample Descriptives}

Descriptive statistics for the continuous study variables are presented in Table 1 . The proportion of participants with lower-level education (high school or lower) was $52.5 \%$ and $47.5 \%$ had a higher level. The proportion of patients with sleep apnea was $26 \%$, with type 2 diabetes $19 \%$, with dyslipidemia $21 \%$, and with hypertension $39 \%$. Except for a significantly larger proportion of type 2 diabetes $\left(\chi^{2}[1, n=80]=7.02, p=0.008\right.$, $\left.p h i=-0.33\right)$ and hypertension $\left(\chi^{2}[1, n=80]=4.97, p=0.026\right.$, $\left.p h i=-0.28\right)$ in males, the presence of medical comorbidities did not differ significantly according to gender. There were also no significant gender differences for any other study variables. The sample means for Vocabulary, Matrix Reasoning, CWIT total error inhibition trial (CWIT error), CWIT total time inhibition trial (CWIT time), and SWM were all in the normal range.

Analysis of Covariance

A series of $2 \times 2$ between-groups analysis of covariance indicated that there was a statistically significant main effect of type 2 diabetes on response inhibition (SSRT), $F(1,76)=$ 8.31, $p=0.005$ (partial $\eta^{2}=0.10$ ), where the latencies of the patient group with type 2 diabetes were longer (mean 234.7; SD 39.3) than in those without it (mean 191.7; SD 44). There was a statistically significant main effect of dyslipidemia on planning (OTS), $F(1$, 74 ) $=4.15, p=0.045$ (partial $\eta^{2}=0.05$ ), where the scores of the patient group with dyslip- 
Walø-Syversen et al.: Bariatric Surgery Candidates: Executive Function, Eating Behavior, and Preoperative WL

Table 2. Study variable correlations

\begin{tabular}{|c|c|c|c|c|c|c|c|c|c|c|c|c|c|c|}
\hline & 1 & 2 & 3 & 4 & 5 & 6 & 7 & 8 & $9 a$ & $9 b$ & 10 & 11 & 12 & 13 \\
\hline 1. BMI at test & 1 & & & & & & & & & & & & & \\
\hline 2. 30-Day preoperative WL (\%) & 0.106 & 1 & & & & & & & & & & & & \\
\hline $\begin{array}{l}\text { 3. Dietary adherence } \\
\text { (mean score) }\end{array}$ & 0.011 & -0.139 & 1 & & & & & & & & & & & \\
\hline $\begin{array}{l}\text { 4. Grazing } \\
\text { (Rep[eat] total score) }\end{array}$ & 0.073 & $-0.249 *$ & -0.190 & 1 & & & & & & & & & & \\
\hline $\begin{array}{l}\text { 5. LOC binge-eating } \\
\text { (frequency) }\end{array}$ & -0.130 & -0.087 & -0.075 & $0.391^{* *}$ & 1 & & & & & & & & & \\
\hline $\begin{array}{l}\text { 6. Working memory } \\
\text { (SWM errors) }\end{array}$ & -0.166 & $-0.293^{* *}$ & 0.003 & 0.090 & 0.009 & 1 & & & & & & & & \\
\hline $\begin{array}{l}\text { 7. Planning } \\
\text { (OTS number of problems) }\end{array}$ & 0.125 & 0.167 & 0.113 & -0.173 & -0.024 & $-0.538^{* *}$ & 1 & & & & & & & \\
\hline $\begin{array}{l}\text { 8. Response Inhibition } \\
\text { (SSRT stop latency) }\end{array}$ & 0.108 & -0.152 & -0.141 & 0.120 & 0.035 & -0.197 & -0.096 & 1 & & & & & & \\
\hline \multicolumn{15}{|l|}{ 9. Inhibitory control (CWIT) } \\
\hline a. Total error inhibition trial & -0.106 & 0.040 & $0.274^{*}$ & -0.014 & -0.039 & -0.104 & 0.013 & $0.362^{* *}$ & 1 & & & & & \\
\hline b. Total time inhibition trial & -0.024 & -0.051 & 0.146 & -0.009 & -0.056 & -0.133 & 0.095 & -0.134 & $0.225^{*}$ & 1 & & & & \\
\hline 10. Age & -0.166 & -0.106 & 0.185 & -0.074 & -0.219 & $-0.269 *$ & -0.085 & $0.222^{*}$ & -0.74 & 0.191 & 1 & & & \\
\hline 11. Matrix reasoning & -0.008 & 0.103 & 0.087 & 0.001 & -0.016 & $-0.276^{*}$ & $0.360^{*}$ & $-0.231^{*}$ & 0.061 & -0.025 & -0.103 & 1 & & \\
\hline 12. Vocabulary & 0.185 & -0.064 & 0.067 & -0.012 & -0.119 & $-0.314^{*}$ & $0.249^{*}$ & -0.068 & 0.081 & $0.337^{*}$ & 0.067 & $0.246^{*}$ & 1 & \\
\hline 13. Depression & -0.62 & $-0.249 *$ & -0.086 & $0.440^{* *}$ & 0.171 & 0.088 & $-0.317^{*}$ & 0.117 & $-0.236^{*}$ & 0.031 & 0.006 & $-0.233^{*}$ & -0.028 & 1 \\
\hline
\end{tabular}

* Correlation is significant at the 0.05 -level. ${ }^{* *}$ Correlation is significant at the 0.01-level. BMI, body mass index; WL, weight loss; LOC, loss-of-control; Rep [eat], repetitive eating questionnaire; SWM, spatial working memory; OTS, One Touch Stockings of Cambridge; SSRT, stop signal reaction time; CWIT, Color-Word Interference Test.

idemia were lower (mean 9.4; SD 2.0) than in the group without it (mean 10.9; SD 2.1). There was a statistically significant main effect of hypertension on inhibitory control (CWIT error), $F(1,75)=4.01, p=0.049$ (partial $\left.\eta^{2}=0.05\right)$, where the scores of the patient group with hypertension were higher (mean 10.84; SD 1.8) than in the group without hypertension (mean 10.3; SD 1.9). There was also a statistically significant main effect of gender on inhibitory control (CWIT time), $F(1,75)=5.11, p=0.026$ (partial $\eta^{2}=0.06$ ), where the scores for females were higher (mean 10.25; SD 2.5) than for males (mean 8.5; SD 3.6). There was no statistically significant main effect of gender or medical comorbidity on any of the other neuropsychological test scores. However, a statistically significant interaction effect between gender and hypertension on working memory (SWM), $F(1,75)=7.24, p=0.009$ (partial $\eta^{2}=0.08$ ), indicated that the effect of hypertension on working memory performance was greater in males.

Hierarchical Multiple Regression

Preliminary bivariate correlations of all variables (Table 2) revealed a significantly small positive correlation $(r$ [80] $=0.274, p<0.05)$ between inhibitory control (CWIT error) and diet adherence, and a significantly small negative correlation $(r$ [80] $=-0.293$, $p<0.01$ ) between working memory (SWM) and 30-day preoperative WL (\%). Post hoc partial correlations showed that the number of dieting days before operation $(r$ [80] = $-0.266, p<0.018)$, initial BMI at referral time $(r[80]=-0.281, p<0.01)$, and the preceding $\%$ WL from referral until testing $(r[80]=-0.303, p<0.007)$ had a marginal effect on the strength of the relationship between working memory and 30-day preoperative WL (\%). Hierarchical regression analyses were used to evaluate the ability of working memory to predict 30-day preoperative WL (\%) while controlling for the influence of gender, age, depression, and IQ (Vocabulary). The initial covariance analyses indicated that hypertension significantly influenced both inhibitory control and working memory, so hypertension was also included as control variable. Control variables explained $9.6 \%$ of the variance in \% WL. Adding working memory (SWM) in Step 2 explained an additional 10.7\% 
Walø-Syversen et al.: Bariatric Surgery Candidates: Executive Function, Eating Behavior, and Preoperative WL

Table 3. Hierarchical multiple regression analysis for prediction of 30-day preoperative WL (\%) and dietary adherence

\begin{tabular}{|c|c|c|c|c|c|c|c|c|c|c|c|c|}
\hline & \multicolumn{6}{|l|}{ Step 1} & \multicolumn{6}{|l|}{ Step 2} \\
\hline & $B(\mathrm{SD})$ & $\beta$ & $r^{2}$ & $\Delta r^{2}$ & $\Delta F$ & $p$ & $B(\mathrm{SD})$ & $\beta$ & $r^{2}$ & $\Delta r^{2}$ & $\Delta F$ & $p$ \\
\hline \multicolumn{13}{|c|}{ 30-day preoperative WL (\%) } \\
\hline & & & 0.096 & 0.096 & 1.57 & 0.178 & & & 0.203 & 0.107 & 9.7 & 0.003 \\
\hline Gender & $-0.401(0.799)$ & -0.059 & & & & 0.618 & $-0.418(0.756)$ & -0.061 & & & & 0.582 \\
\hline Age & $-0.021(0.031)$ & -0.079 & & & & 0.490 & $0.007(0.031)$ & 0.026 & & & & 0.817 \\
\hline Vocabulary & $-0.234(0.183)$ & -0.145 & & & & 0.205 & $-0.426(0.184)$ & -0.263 & & & & 0.023 \\
\hline Depression & $-0.232(0.097)$ & -0.267 & & & & 0.020 & $-0.219(0.092)$ & -0.252 & & & & 0.020 \\
\hline Hypertension & $0.425(0.698)$ & 0.071 & & & & 0.544 & $0.453(0.660)$ & 0.076 & & & & 0.494 \\
\hline Working memory & & & & & & & $-0.052(0.017)$ & -0.362 & & & & 0.003 \\
\hline \multicolumn{13}{|l|}{ Dietary adherence } \\
\hline & & & 0.043 & 0.043 & 0.844 & 0.502 & & & 0.114 & 0.071 & 5.932 & 0.017 \\
\hline Gender & $-0.394(0.344)$ & -0.135 & & & & 0.256 & $-0.328(0.334)$ & -0.134 & & & & 0.329 \\
\hline Vocabulary & $0.040(0.079)$ & 0.058 & & & & 0.617 & $0.033(0.077)$ & 0.048 & & & & 0.669 \\
\hline Depression & $-0.033(0.042)$ & -0.089 & & & & 0.443 & $-0.010(0.042)$ & -0.027 & & & & 0.814 \\
\hline Hypertension & $-0.195(0.301)$ & -0.077 & & & & 0.519 & $-0.300(0.294)$ & -0.118 & & & & 0.311 \\
\hline Inhibitory control & & & & & & & $0.183(0.075)$ & 0.278 & & & & 0.017 \\
\hline
\end{tabular}

WL, weight loss; SD, standard deviation.

of the variance in \% WL. It was found that working memory (the number of SWM errors) significantly predicted $\%$ WL $(\beta=-0.362, p=0.003)$. The final whole model was significant: $F(6,73)=3.09, p=0.009$. A subsequent hierarchical regression analysis was used to assess the ability of inhibitory control to predict dietary adherence, after controlling for gender, depression, IQ (Vocabulary), and hypertension (Table 3). The control variables entered at Step 1 explained $4.3 \%$ of the variance in dietary adherence. Adding inhibitory control in Step 2 explained an additional $7.1 \%$ of the variance in adherence to dietary recommendations. It was found that inhibitory control (CWIT error) significantly predicted dietary adherence $(\beta=0.278, p=0.017)$. The final model was not significant: $F(5,74)=1.90, p=$ 0.103 .

\section{Discussion}

This study aimed to examine the relationship between executive function, BMI, preoperative WL and eating behavior in a sample of patients presenting for bariatric surgery. We assumed that executive function would be related to preoperative BMI and WL. We also expected executive function to predict healthier eating patterns, i.e., greater adherence to dietary recommendations, and less eating pathology, i.e., less grazing behavior and fewer LOC binge-eating episodes. We also examined the impact of medical comorbidities and depression on neuropsychological test performance. To sum up, our main findings were that better working memory predicted WL in the 30 days prior to surgery and that 1 measure of inhibitory control predicted self-reported adherence to dietary recommendations before the required WL period ( 4 weeks prior to neuropsychological testing and prior to the obligatory low-calorie diet). Furthermore, the results indicated that somatic comorbidity and depression had a moderate influence on neuropsychological test results and that there were few differences due to gender. Contrary to our expectations, the executive function measures were not associated with self-reported LOC binge-eating, grazing, or baseline BMI.

\section{Somatic Comorbidity}

Overall, males had greater medical comorbidity than females, but medical comorbidity did not seem to have a very strong influence on neuropsychological performance. This may 
be explained by patients being under current medical treatment and not presenting with "active" and untreated disease. Besides hypertension, both type 2 diabetes and dyslipidemia had an effect on working memory (OTS) and inhibitory control (SSRT), but interpreting the significance of these findings presents a challenge. The effect sizes were generally small to moderate, and the analyses were not adjusted for multiple comparisons or unevenly sized groups, which may have increased the risk of a type 1 error.

\section{Working Memory and Inhibitory Control}

We found that working memory capacity predicted WL during the 4 weeks prior to surgery. It has been proposed that working memory contributes to the ability to subdue information (and downregulate desires) which conflicts with a long-term goal [8]. Two recent reviews $[5,8]$ proposed that working memory is linked to self-monitoring and initiating healthy choices [5], i.e., greater consumption of fruit and vegetables, planning meals, calorie-tracking, and weighing. With these points as a backdrop, our findings seem in line with the previous literature, i.e., that those with a better working memory lost more weight prior to surgery. However, our study design could not disentangle the behavioral process by which working memory functions to obtain WL. We found that inhibitory control, as assessed by error performance in the inhibition condition in the D-KEFS CWIT, predicted self-reported adherence to dietary recommendations, such that those with fewer errors reported a higher dietary adherence. Dietary recommendations focused on the overall intake of various food groups, such as limiting the intake of sugar and fat, choosing dairy and meat products with less fat, and eating 5 portions of fruit per day. This finding is not directly comparable with earlier studies, since test formats and outcome measures both vary [9]. We used "time to complete" and "number of errors" as outcome measures, and only the latter was related to dietary adherence. As most participants performed in the normal range regarding time completion, this may indicate that error performance was a more sensitive measure of inhibition in our sample, i.e., participants worked equally quickly, but for some at the expense of not being able to inhibit the incorrect response [56]. Although inhibitory control predicted merely a minor proportion of dietary adherence, our results indicate that inhibitory control may indeed be involved in dietary adherence before surgery.

\section{Additional Findings}

The executive function measures showed no relationship to self-reported LOC bingeeating, grazing, or BMI. Contrary to our hypothesis, we did not find any indication of a relationship between the frequency of LOC binge-eating episodes and inhibitory control (D-KEFS CWIT) in our sample. The proportion of participants reported having experienced at least 1 episode of LOC binge-eating was $20 \%$. However, only $11 \%$ ( 9 patients) reported having $>4$ episodes, which may be indicative of clinical pathology. This low presence of binge-eating would probably exclude the detection of any relationship to executive function deficits. Unexpectedly, response inhibition (SSRT) was not related to eating behavior. In earlier studies, SSRT separated participants with obesity from lean controls [20] and was especially indicative of group differences in experimental designs using food-specific stimuli [9]. In line with the reasoning that severe obesity is only related to impaired food-specific inhibitory control [57], our results are less surprising. However, patients' understating of their eating pathology may also conceivably explain these findings. Contrary to our expectations, BMI did not correlate with executive function performance or any other study variables. This may be somewhat surprising when considering previous findings $[23,58]$. However, in addition to the possibility of patients underreporting, it is possible that the BMI range in our sample was too restricted to allow for any statistically significant relationships. Dietary adherence was 
Walø-Syversen et al.: Bariatric Surgery Candidates: Executive Function, Eating Behavior, and Preoperative WL

not associated with WL or other measures of eating behavior; however, the dietary adherence items reflected the national dietary guidelines. As such, this set of questions was not a specific measure of presurgery diet adherence. It is also likely that they were too general to relate to eating pathology or capture eating behavior that would lead to WL in a very short time. Regarding depression, we found that it correlated with several study variables. It was moderately related to planning (OTS) and grazing, and predicted WL in our multiple hierarchical regression model. These results confirm the value of controlling for and taking depression into consideration in clinical studies. The relationship between grazing and depression deserves further investigation.

\section{Strengths and Limitations}

The main strength of this study is the use of several sensitive, precise, and objective performance-based measures of executive function as well as selected self-report measures that encompass diverse but important clinical aspects of eating patterns in bariatric surgery patients. The study has limitations. First, the sample size was somewhat limited. Second, it was an exploratory correlational study done in a clinical setting with no control group, which limits the overview of potential confounding factors and conclusions of causality. However, we reported the baseline data collection from a longitudinal project with a followup period of up to 5 years, during which patients serve as their own controls over time. Third, we could have used a more exact and validated measurement of dietary adherence and included measures on eating behavior less influenced by common self-report bias like underreporting. Last, both self-selection to participate in the study and underreporting may limit the generalizability of our findings. For instance, patients' self-reports indicated that the study sample had a lower reported psychopathology than is often reported in bariatric samples [41]. This may be due to the aforementioned issue of restricted sampling yielding a less symptomatic and diverse sample or else to underreporting. Regarding the latter, self-reports were delivered long after surgery was decided upon. We also underscored anonymity and that responses would have no effect on further treatment, hoping to promote correct disclosure.

\section{Conclusions}

In spite of some limitations, our study suggests that executive function is a contributor to eating behavior and WL in the preoperative period. Many measures yielded nonsignificant results and effect sizes were small to moderate, yet the findings indicated that inhibitory control was related to adherence to dietary recommendations and working memory predicted WL in the 30 days prior to surgery. These results suggest that executive functioning is involved in the change processes taking place prior to surgery, and it therefore merits further investigation. Our findings need confirmation and further studies are required to establish the clinical significance of executive function before bariatric surgery.

\section{Acknowledgements}

We would like to express our appreciation to the surgeons at the Department of Morbid Obesity and Bariatric Surgery, Oslo University Hospital, Oslo, Norway, for recruiting patients for the study. We also thank administrator Marianne Sæter for retrieving data from patient medical records. 
Walø-Syversen et al.: Bariatric Surgery Candidates: Executive Function, Eating Behavior, and Preoperative WL

\section{Statement of Ethics}

Participants received oral and written information about the study before giving their written consent to participate. Study approval was given by the Regional Committee for Medical and Health Research Ethics (2015/402) of South-Eastern Norway and the Privacy Ombudsman for Research at the Oslo University Hospital.

\section{Disclosure Statement}

The authors have no conflicts of interest to declare.

\section{Funding Sources}

This work was supported by a grant from the Health Region South-East $(027,2017)$.

\section{References}

1 Prickett C, Brennan L, Stolwyk R. Examining the relationship between obesity and cognitive function: a systematic literature review. Obes Res Clin Pract. 2015 Mar-Apr;9(2):93-113.

2 Spitznagel MB, Hawkins M, Alosco M, Galioto R, Garcia S, Miller L, et al. Neurocognitive Effects of Obesity and Bariatric Surgery. Eur Eat Disord Rev. 2015 Nov;23(6):488-95.

3 Smith E, Hay P, Campbell L, Trollor JN. A review of the association between obesity and cognitive function across the lifespan: implications for novel approaches to prevention and treatment. Obes Rev.2011 Sep;12(9): 740-55.

4 Gunstad J, Strain G, Devlin MJ, Wing R, Cohen RA, Paul RH, et al. Improved memory function 12 weeks after bariatric surgery. Surg Obes Relat Dis. 2011 Jul-Aug;7(4):465-72.

5 Gettens KM, Gorin AA. Executive function in weight loss and weight loss maintenance: a conceptual review and novel neuropsychological model of weight control. J Behav Med. 2017 Oct;40(5):687-701.

6 Fitzpatrick S, Gilbert S, Serpell L. Systematic review: are overweight and obese individuals impaired on behavioural tasks of executive functioning? Neuropsychol Rev. 2013 Jun;23(2):138-56.

7 Galioto R, Gunstad J, Heinberg LJ, Spitznagel MB. Adherence and weight loss outcomes in bariatric surgery: does cognitive function play a role? Obes Surg. 2013 Oct;23(10):1703-10.

8 Dohle S, Diel K, Hofmann W. Executive functions and the self-regulation of eating behavior: a review. Appetite. 2018 May 1;124:4-9.

9 Lavagnino L, Arnone D, Cao B, Soares JC, Selvaraj S. Inhibitory control in obesity and binge eating disorder: A systematic review and meta-analysis of neurocognitive and neuroimaging studies. Neurosci Biobehav Rev. 2016 Sep;68:714-26.

10 Spitznagel MB, Galioto R, Limbach K, Gunstad J, Heinberg L. Cognitive function is linked to adherence to bariatric postoperative guidelines. Surg Obes Relat Dis. 2013 Jul-Aug; 9(4):580-5.

11 Galioto R, Bond D, Gunstad J, Pera V, Rathier L, Tremont G. Executive functions predict weight loss in a medically supervised weight loss programme. Obes Sci Pract. 2016 Dec;2(4):334-40.

12 Kulendran M, Vlaev I, Sugden C, King D, Ashrafian H, Gately P, et al. Neuropsychological assessment as a predictor of weight loss in obese adolescents. Int J Obes. 2014 Apr;38(4):507-12.

13 Spitznagel MB, Garcia S, Miller LA, Strain G, Devlin M, Wing R, et al. Cognitive function predicts weight loss after bariatric surgery. Surg Obes Relat Dis. 2013 May-Jun;9(3):453-9.

14 Spitznagel MB, Alosco M, Strain G, Devlin M, Cohen R, Paul R, et al. Cognitive function predicts 24-month weight loss success after bariatric surgery. Surg Obes Relat Dis. 2013 Sep-Oct; 9(5):765-70.

15 Kulendran M, Borovoi L, Purkayastha S, Darzi A, Vlaev I. Impulsivity predicts weight loss after obesity surgery. Surg Obes Relat Dis. 2017 Jun;13(6):1033-40.

16 Lezak MD, editor. Neuropsychological Assessment. 5th ed. Oxford: University Press; 2012.

17 Diamond A. Executive functions. Annu Rev Psychol. 2013;64(1):135-68.

18 Miyake A, et al. The unity and diversity of executive functions and their contributions to complex frontal lobe tasks: a latent variable analysis. Cogn Psychol; 2000 Aug;41(1):49-100.

19 Stanek KM, Strain G, Devlin M, Cohen R, Paul R, Crosby RD, et al. Body mass index and neurocognitive functioning across the adult lifespan. Neuropsychology. 2013 Mar;27(2):141-51.

20 Bartholdy S, Dalton B, O'Daly OG, Campbell IC, Schmidt U. A systematic review of the relationship between eating, weight and inhibitory control using the stop signal task. Neurosci Biobehav Rev. 2016 May;64:35-62. 
Walø-Syversen et al.: Bariatric Surgery Candidates: Executive Function, Eating Behavior, and Preoperative WL

21 Reinert KR, Po'e EK, Barkin SL. The relationship between executive function and obesity in children and adolescents: a systematic literature review. J Obes. 2013;2013:820956.

22 Gunstad J, Lhotsky A, Wendell CR, Ferrucci L, Zonderman AB. Longitudinal examination of obesity and cognitive function: results from the Baltimore longitudinal study of aging. Neuroepidemiology. 2010;34(4): 222-9.

23 Gunstad J, Paul RH, Cohen RA, Tate DF, Spitznagel MB, Gordon E. Elevated body mass index is associated with executive dysfunction in otherwise healthy adults. Compr Psychiatry. 2007 Jan-Feb;48(1):57-61.

24 Wolf PA, Beiser A, Elias MF, Au R, Vasan RS, Seshadri S. Relation of obesity to cognitive function: importance of central obesity and synergistic influence of concomitant hypertension. The Framingham Heart Study. Curr Alzheimer Res. 2007 Apr;4(2):111-6.

25 Kulendran M, et al. The Role of Impulsivity in Obesity as Measured by Inhibitory Control: A Systematic Review. Med Res Arch. 2017;5(8).

26 Maayan L, Hoogendoorn C, Sweat V, Convit A. Disinhibited eating in obese adolescents is associated with orbitofrontal volume reductions and executive dysfunction. Obesity (Silver Spring). 2011 Jul;19(7):1382-7.

27 Coppin G, Nolan-Poupart S, Jones-Gotman M, Small DM. Working memory and reward association learning impairments in obesity. Neuropsychologia. 2014 Dec;65:146-55.

28 Boeka AG, Lokken KL. Neuropsychological performance of a clinical sample of extremely obese individuals. Arch Clin Neuropsychol. 2008 Jul;23(4):467-74.

29 Whitelock V, Nouwen A, van den Akker O, Higgs S. The role of working memory sub-components in food choice and dieting success. Appetite. 2018 May;124:24-32.

30 Wyckoff EP, Evans BC, Manasse SM, Butryn ML, Forman EM. Executive functioning and dietary intake: neurocognitive correlates of fruit, vegetable, and saturated fat intake in adults with obesity. Appetite. 2017 Apr; 111 : 79-85.

31 Manasse SM, Juarascio AS, Forman EM, Berner LA, Butryn ML, Ruocco AC. Executive functioning in overweight individuals with and without loss-of-control eating. Eur Eat Disord Rev. 2014 Sep;22(5):373-7.

32 Brockmeyer T, Hamze Sinno M, Skunde M, Wu M, Woehning A, Rudofsky G, et al. Inhibitory Control and Hedonic Response towards Food Interactively Predict Success in a Weight Loss Programme for Adults with Obesity. Obes Facts. 2016;9(5):299-309.

33 Colles SL, Dixon JB, O’Brien PE. Grazing and loss of control related to eating: two high-risk factors following bariatric surgery. Obesity (Silver Spring). 2008 Mar;16(3):615-22.

34 Conceição EM, Mitchell JE, Engel SG, Machado PP, Lancaster K, Wonderlich SA. What is "grazing"? Reviewing its definition, frequency, clinical characteristics, and impact on bariatric surgery outcomes, and proposing a standardized definition. Surg Obes Relat Dis. 2014 Sep-Oct;10(5):973-82.

35 Conceição EM, Mitchell JE, Machado PP, Vaz AR, Pinto-Bastos A, Ramalho S, et al. Repetitive eating questionnaire [Rep(eat)-Q]: enlightening the concept of grazing and psychometric properties in a Portuguese sample. Appetite. 2017 Oct;117 Suppl C:351-8.

36 Davis C. A commentary on the associations among 'food addiction', binge eating disorder, and obesity: overlapping conditions with idiosyncratic clinical features. Appetite. 2017 Aug 1;115:3-8.

37 Tewksbury C, Williams NN, Dumon KR, Sarwer DB. Preoperative Medical Weight Management in Bariatric Surgery: A Review and Reconsideration. Obes Surg. 2017 Jan;27(1):208-14.

38 Rochette AD, Spitznagel MB, Strain G, Devlin M, Crosby RD, Mitchell JE, et al. Mild cognitive impairment is prevalent in persons with severe obesity. Obesity (Silver Spring). 2016 Jul;24(7):1427-9.

39 Sargénius HL, Lydersen S, Hestad K. Neuropsychological function in individuals with morbid obesity: a crosssectional study. BMC Obes. 2017 Jan;4(1):6.

40 Spitznagel MB, Alosco M, Galioto R, Strain G, Devlin M, Sysko R, et al. The role of cognitive function in postoperative weight loss outcomes: 36-month follow-up. Obes Surg. 2014 Jul;24(7):1078-84.

41 Dawes AJ, Maggard-Gibbons M, Maher AR, Booth MJ, Miake-Lye I, Beroes JM, et al. Mental health conditions among patients seeking and undergoing bariatric surgery: A meta-analysis. JAMA. 2016 Jan;315(2):150-63.

42 Rock PL, Roiser JP, Riedel WJ, Blackwell AD. Cognitive impairment in depression: a systematic review and meta-analysis. Psychol Med. 2014 Jul;44(10):2029-40.

43 Kvalem IL, Bergh I, von Soest T, Rosenvinge JH, Johnsen TA, Martinsen EW, et al. A comparison of behavioral and psychological characteristics of patients opting for surgical and conservative treatment for morbid obesity. BMC Obes. 2016 Feb;3(1):6.

44 Bergh I, Lundin Kvalem I, Risstad H, Sniehotta FF. Preoperative predictors of adherence to dietary and physical activity recommendations and weight loss one year after surgery. Surg Obes Relat Dis. 2016 May;12(4):910-8.

45 Helsedirektoratet. Anbefalinger om kosthold, ernæring og fysisk aktivitet. Helsedirektoratet IS-2170. Oslo: Nasjonalt råd for ernæring; 2012.

46 Fairburn CG. Cognitive behavior therapy and eating disorders. 2009.

47 Rø 0, Reas DL, Lask B. Norms for the Eating Disorder Examination Questionnaire among female university students in Norway. Nord J Psychiatry. 2010 Dec;64(6):428-32.

48 Reas DL, Lindvall Dahlgren C, Wonderlich J, Syversen G, Lundin Kvalem I. Confirmatory factor analysis and psychometric properties of the Norwegian version of the Repetitive Eating Questionnaire: further evidence for two distinct subtypes of grazing behaviour. Eur Eat Disord Rev. 2019 Mar;27(2):205-11.

49 Mykletun A, Stordal E, Dahl AA. Hospital Anxiety and Depression (HAD) scale: factor structure, item analyses and internal consistency in a large population. Br J Psychiatry. 2001 Dec;179(6):540-4. 
Walø-Syversen et al.: Bariatric Surgery Candidates: Executive Function, Eating Behavior, and Preoperative WL

50 Bjelland I, Dahl AA, Haug TT, Neckelmann D. The validity of the Hospital Anxiety and Depression Scale. An updated literature review. J Psychosom Res. 2002 Feb;52(2):69-77.

51 Wechsler D. Wechsler Abbreviated Scale of Intelligence (WASI). San Antonio (TX): NCS Pearson Inc; 1999.

52 Haukeland E. Weschler Adult Intelligence Scale. 4th ed. Norwegian Version. Enschede: Ipskamp Drukkers; 2012.

53 Delis Kaplan Executive System (D-KEFS). Norwegian Manual Supplement. Oslo: Psykologiforlaget; 2005.

54 Sahakian BJ, Morris RG, Evenden JL, Heald A, Levy R, Philpot M, et al. A comparative study of visuospatial memory and learning in Alzheimer-type dementia and Parkinson's disease. Brain. 1988 Jun;111(Pt 3):695718.

55 Verbruggen F, Logan GD. Response inhibition in the stop-signal paradigm. Trends Cogn Sci. 2008 Nov; 12(11): 418-24.

56 Delis DC, Kaplan E, Kramer JH. Delis - Kaplan Executive Function System: Examiners Manual. Psychological Corporation; 2001.

57 Houben K, Nederkoorn C, Jansen A. Eating on impulse: the relation between overweight and food-specific inhibitory control. Obesity (Silver Spring). 2014 May;22(5):E6-8.

58 Volkow ND, Wang GJ, Telang F, Fowler JS, Goldstein RZ, Alia-Klein N, et al. Inverse association between BMI and prefrontal metabolic activity in healthy adults. Obesity (Silver Spring). 2009 Jan;17(1):60-5. 